Korean J. Math. 22 (2014), No. 1, pp. 1-28

http://dx.doi.org/10.11568/kjm.2014.22.1.1

\title{
THE POSITIVITY OF THE HYPERGEOMETRIC TRANSLATION OPERATORS ASSOCIATED TO THE CHEREDNIK OPERATORS AND THE \\ HECKMAN-OPDAM THEORY ATTACHED TO THE ROOT SYSTEMS OF TYPE $B_{2}$ AND $C_{2}$
}

\author{
Khalifa Trimèche
}

\begin{abstract}
We consider the hypergeometric translation operator associated to the Cherednik operators and the Heckman-Opdam theory attached to the root system of type $B_{2}$. We prove in this paper that these operators are positivity preserving and allow positive integral representations. In particular we deduce that the product formulas of the Opdam-Cherednik and the Heckman-Opdam kernels are positive integral transforms, and we obtain best estimates of these kernels.

The method used to obtain the previous results shows that these results are also true in the case of the root system of type $C_{2}$.
\end{abstract}

\section{Introduction}

In [1], Cherednik introduced a family of differential-difference operators that nowadays bear his name. These operators play a crucial role in

Received February 6, 2013. Revised March 13, 2013. Accepted March 13, 2013.

2010 Mathematics Subject Classification: 33E30, 33C67, 51F15.

Key words and phrases: Cherednik operators, Heckman-Opdam theory, Root systems of type $B_{2}$ and $C_{2}$, hypergeometric translation operators, Opdam-Cherednik kernel, Heckman-Opdam kernel, product formula.

This research was supported by Incheon National University Research Grant, 2013-2014.

(c) The Kangwon-Kyungki Mathematical Society, 2014.

This is an Open Access article distributed under the terms of the Creative commons Attribution Non-Commercial License (http://creativecommons.org/licenses/by -nc/3.0/) which permits unrestricted non-commercial use, distribution and reproduction in any medium, provided the original work is properly cited. 
the theory of Heckman-Opdam's hypergeometric functions, which generalize the theory of Harish-Chandra's spherical functions on Riemannian symmetric spaces (see $[2,3,5])$.

To study in $[7,8]$ a harmonic analysis associated with the Cherednik operators and the Heckman-Opdam theory, the author has introduced the hypergeometric translation operators. In many situations to solve problems of this harmonic analysis we need the positivity of these operators, and the product formulas of the Opdam-Cherednik and the Heckman-Opdam kernels are given by integrals with positive measures. These properties are not yet proved in the general case, they are obtained only in the one dimensional case (see [9]), and in the multidimensional case under some conditions on the root system and the multiplicity function (see [10]).

This paper is a contribution towards these questions in the case of the Cherednik operators and the Heckman-Opdam theory attached to the root system of type $B_{2}$.

We prove in this paper the positivity of the hypergeometric translation operators $\mathcal{T}_{x}, \mathcal{T}_{x}^{W}, x \in \mathbb{R}^{2}$, associated respectively to the Cherednik operators and the Heckman-Opdam theory, and we deduce that for all $x, u \in \mathbb{R}^{2}$, there exist positive measures $m_{x, u}, m_{x, u}^{W}$ on $\mathbb{R}^{2}$ with compact support and of norm equal to 1 , such that

- For all $C^{\infty}$-function $g$ on $\mathbb{R}^{2}$ we have

$$
\mathcal{T}_{x}(g)(u)=\int_{\mathbb{R}^{2}} g(z) d m_{x, u}(z) .
$$

- For all $C^{\infty}$-function $g$ on $\mathbb{R}^{2}$ invariant by the Weyl group $W$, we have

$$
\mathcal{T}_{x}^{W}(g)(u)=\int_{\mathbb{R}^{2}} g(z) d m_{x, u}^{W}(z) .
$$

From the relations (1), (2) we deduce the following product formulas for the Opdam-Cherednik kernel $G_{\lambda}(x), \lambda \in \mathbb{C}^{2}$, and the Heckman-Opdam kernel $F_{\lambda}(x), \lambda \in \mathbb{C}^{2}$ :

$$
\begin{aligned}
& \forall x, u \in \mathbb{R}^{2}, G_{\lambda}(x) G_{\lambda}(u)=\int_{\mathbb{R}^{2}} G_{\lambda}(z) d m_{x, u}(z), \\
& \forall x, u \in \mathbb{R}^{2}, F_{\lambda}(x) F_{\lambda}(u)=\int_{\mathbb{R}^{2}} F_{\lambda}(z) d m_{x, u}^{W}(z) .
\end{aligned}
$$


These formulas imply the following estimates for the functions $G_{\lambda}(x)$ and $F_{\lambda}(x)$ :

$$
\begin{aligned}
& \forall x, \in \mathbb{R}^{2}, \forall \lambda \in \mathbb{R}^{2},\left|G_{\lambda}(x)\right| \leq 1, \\
& \forall x, \in \mathbb{R}^{2}, \forall \lambda \in \mathbb{R}^{2},\left|F_{\lambda}(x)\right| \leq 1 .
\end{aligned}
$$

Our proof of the positivity of the hypergeometric translation operator $\mathcal{T}_{x}, x \in \mathbb{R}^{2}$, uses essentially the properties of the heat kernel $p_{t}(x, y), t>$ 0 , associated with the Cherednik operators and the positivity of the transmutation operators associated with the Cherednik operators proved in [11]. We deduce the positivity of the hypergeometric translation operator $\mathcal{T}_{x}^{W}, x \in \mathbb{R}^{2}$, from the previous result and the relation between $\mathcal{T}_{x}$ and $\mathcal{T}_{x}^{W}$

We remark that the method employed in this paper can be applied to the Jacobi-Cherednik operator on $\mathbb{R}$, and to the Cherednik operators and the Heckman-Opdam theory attached to the root system of type $C_{2}$.

\section{The Cherednik operators on $\mathbb{R}^{2}$ and their eigenfunctions}

We consider $\mathbb{R}^{2}$ with the standard basis $\left\{e_{1}, e_{2}\right\}$ and inner product $\langle.,$.$\rangle for which this basis is orthonormal. We extend this inner product$ to a complex bilinear form on $\mathbb{C}^{2}$.

2.1. The root system of type $B_{2}$ and the multiplicity function. The root system of type $B_{2}$ can be identified with the set $\mathcal{R}$ given by

$$
\mathcal{R}=\left\{ \pm e_{1}, \pm e_{2}\right\} \cup\left\{ \pm e_{1} \pm e_{2}\right\},
$$

which can also be written in the form

$$
\mathcal{R}=\left\{ \pm \alpha_{1}, \pm \alpha_{2}, \pm \alpha_{3} \pm \alpha_{4}\right\}
$$

with

$$
\alpha_{1}=e_{1}, \alpha_{2}=e_{2}, \alpha_{3}=\left(e_{1}-e_{2}\right), \alpha_{4}=\left(e_{1}+e_{2}\right) .
$$

We denote by $\mathcal{R}_{+}$the set of positive roots

$$
\mathcal{R}_{+}=\left\{\alpha_{1}, \alpha_{2}, \alpha_{3}, \alpha_{4}\right\}
$$

and by $\mathcal{R}_{+}^{o}$ the set of positive indivisible roots i.e, the roots $\alpha \in \mathcal{R}_{+}$such that $\frac{\alpha}{2} \notin \mathcal{R}_{+}$. Then we have

$$
\mathcal{R}_{+}^{0}=\mathcal{R}_{+} .
$$


For $\alpha \in \mathcal{R}$, we consider

$$
r_{\alpha}(x)=x-\langle\breve{\alpha}, x\rangle \alpha, \text { with } \breve{\alpha}=\frac{2 \alpha}{\|\alpha\|^{2}},
$$

the reflection in the hyperplan $H_{\alpha} \subset \mathbb{R}^{2}$ orthogonal to $\alpha$. The reflections $r_{\alpha}, \alpha \in \mathcal{R}$, generate a finite group $W \subset O(2)$, called the Weyl group associated with $\mathcal{R}$. In this case $W$ is isomorphic to the hyperoctahedral group which is generated by permutations and sign changes of the $e_{i}, i=$ 1,2 ,

The multiplicity function $k: \mathcal{R} \rightarrow] 0,+\infty[$ can be written in the form $k=\left(k_{1}, k_{2}\right)$ where $k_{1}$ is the value on the roots $\alpha_{1}, \alpha_{2}$, and $k_{2}$ is the value on the roots $\alpha_{3}, \alpha_{4}$.

The positive Weyl chamber denoted by $\mathfrak{a}^{+}$is given by

$$
\mathfrak{a}^{+}=\left\{x \in \mathbb{R}^{2} ; \forall \alpha \in \mathcal{R}_{+},\langle\alpha, x\rangle>0\right\},
$$

it can also be written in the form

$$
\mathfrak{a}^{+}=\left\{\left(x_{1}, x_{2}\right) \in \mathbb{R}^{2} ; x_{1}>x_{2}>0\right\},
$$

we denote by $\overline{\mathfrak{a}^{+}}$its closure. Let also $\mathbb{R}_{\text {reg }}^{2}$ be the subset of regular elements in $\mathbb{R}^{2}$, i.e., those elements which belong to no hyperplane $H_{\alpha}=$ $\left\{x \in \mathbb{R}^{2} ;\langle\alpha, x\rangle=0\right\}, \alpha \in \mathcal{R}$.

Let $\mathcal{A}_{k}$ denote the weight function

$$
\forall x \in \mathbb{R}^{2}, \mathcal{A}_{k}(x)=\prod_{\alpha \in \mathcal{R}_{+}}\left|\sinh \left\langle\frac{\alpha}{2}, x\right\rangle\right|^{2 k(\alpha)} .
$$

REMARK 2.1. The root system of type $C_{2}$ can be identified with the set $\mathcal{R}$ given by

$$
\mathcal{R}=\left\{ \pm 2 e_{1}, \pm 2 e_{2}\right\} \cup\left\{ \pm e_{1} \pm e_{2}\right\},
$$

which can also be written in the form

$$
\mathcal{R}=\left\{ \pm \alpha_{1}, \pm \alpha_{2}, \pm \alpha_{3}, \pm \alpha_{4}\right\},
$$

with

$$
\alpha_{1}=2 e_{1}, \alpha_{2}=2 e_{2}, \alpha_{3}=\left(e_{1}-e_{2}\right), \alpha_{4}=\left(e_{1}+e_{2}\right) .
$$

The set of positive roots is the following

$$
\mathcal{R}_{+}=\left\{\alpha_{1}, \alpha_{2}, \alpha_{3}, \alpha_{4}\right\}
$$

If we denote by $W\left(C_{2}\right)$ the Weyl group associated to the root system $\mathcal{R}$ of type $C_{2}$, then we have

$$
W\left(C_{2}\right)=W\left(B_{2}\right) .
$$


We denote also by $k=\left(k_{1}, k_{2}\right)$ the multiplicity function of the root system $\mathcal{R}$ of $C_{2}$, where $k_{1}$ is the value on the roots $\alpha_{1}, \alpha_{2}$, and $k_{2}$ is the value on the roots $\alpha_{3}, \alpha_{4}$.

In the remainder of the paper we shall give the results and their proofs only for the root system of type $B_{2}$. It is easy to obtain the analogous of these results in the case of the root system of type $C_{2}$.

2.2. The Cherednik operators on $\mathbb{R}^{2}$. The Cherednik operators $T_{j}, j=1,2$, on $\mathbb{R}^{2}$ associated with the Weyl group $W$ and the multiplicity function $k$ are defined for $f$ of class $C^{1}$ on $\mathbb{R}^{2}$ and $x \in \mathbb{R}_{\text {reg }}=\mathbb{R}^{2} \backslash \bigcup_{\alpha \in \mathcal{R}} H_{\alpha}$ by

$$
T_{j} f(x)=\frac{\partial}{\partial x_{j}} f(x)+\sum_{\alpha \in \mathcal{R}_{+}} \frac{k(\alpha) \alpha^{j}}{1-e^{-\langle\alpha, x\rangle}}\left\{f(x)-f\left(r_{\alpha} x\right)\right\}-\rho_{j} f(x),
$$

with

$$
\rho_{j}=\frac{1}{2} \sum_{\alpha \in \mathcal{R}_{+}} k(\alpha) \alpha^{j}, \quad \text { and } \alpha^{j}=\left\langle\alpha, e_{j}\right\rangle .
$$

These operators can also be written in the following form

$$
\begin{aligned}
T_{j} f(x) & =\frac{\partial}{\partial x_{1}} f(x)+k_{1} \frac{\left\{f(x)-f\left(r_{\alpha_{1}} x\right)\right\}}{1-e^{-\left\langle\alpha_{1}, x\right\rangle}}+k_{2}\left[\frac{f(x)-f\left(r_{\alpha_{3}} x\right)}{1-e^{-\left\langle\alpha_{3}, x\right\rangle}}\right. \\
& \left.+\frac{f(x)-f\left(r_{\alpha_{4}} x\right)}{1-e^{-\left\langle\alpha_{4}, x\right\rangle}}\right]-\left(\frac{1}{2} k_{1}+k_{2}\right) f(x) . \\
T_{2} f(x) & =\frac{\partial}{\partial x_{2}} f(x)+k_{1} \frac{\left\{f(x)-f\left(r_{\alpha_{2}} x\right)\right\}}{1-e^{-\left\langle\alpha_{2}, x\right\rangle}} \\
& +k_{2}\left[-\frac{f(x)-f\left(r_{\alpha_{3}} x\right)}{1-e^{-\left\langle\alpha_{3}, x\right\rangle}}+\frac{f(x)-f\left(r_{\alpha_{4}} x\right)}{1-e^{-\left\langle\alpha_{4}, x\right\rangle}}\right]-\frac{1}{2} k_{1} f(x) .
\end{aligned}
$$

2.3. The eigenfunctions of the Cherednik operators. We denote by $G_{\lambda}, \lambda \in \mathbb{C}^{2}$, the eigenfunction of the operators $T_{j}, j=1,2$. It is the unique analytic function on $\mathbb{R}^{2}$ which satisfies the differential difference system

$$
\begin{cases}T_{j} G_{\lambda}(x) & =-i \lambda_{j} G_{\lambda}(x), x \in \mathbb{R}^{2}, j=1,2, \\ G_{\lambda}(0) & =1\end{cases}
$$

It is called the Opdam-Cherednik kernel. 
We consider the function $F_{\lambda}, \lambda \in \mathbb{C}^{2}$, defined by

$$
\forall x \in \mathbb{R}^{2}, F_{\lambda}(x)=\frac{1}{|W|} \sum_{w \in W} G_{\lambda}(w x) .
$$

This function is the unique analytic $W$-invariant function on $\mathbb{R}^{2}$, which satisfies the partial differential equation

$$
\begin{cases}p(T) F_{\lambda}(x) & =p(-i \lambda) F_{\lambda}(x), \quad x \in \mathbb{R}^{2}, \\ F_{\lambda}(0) & =1\end{cases}
$$

for all $W$-invariant polynomials $p$ on $\mathbb{R}^{2}$ and $p(T)=p\left(T_{1}, T_{2}\right)$. It is called the Heckman-Opdam kernel.

The functions $G_{\lambda}$ and $F_{\lambda}$ possess the following properties

i) For all $x \in \mathbb{R}^{2}$ the function $\lambda \rightarrow G_{\lambda}(x)$ is entire on $\mathbb{C}^{2}$.

ii) We have

$$
\forall x \in \mathbb{R}^{2}, \forall \lambda \in \mathbb{C}^{2}, \overline{G_{\lambda}(x)}=G_{-\bar{\lambda}}(x) .
$$

iii) We have

$$
\forall x \in \mathbb{R}^{2}, \forall \lambda \in \mathbb{C}^{2},\left|G_{\lambda}(x)\right| \leq G_{i \operatorname{Im}(\lambda)}(x) .
$$

iv) We have

$$
\begin{aligned}
& \forall x \in \mathbb{R}^{2}, \forall \lambda \in \mathbb{R}^{2},\left|G_{\lambda}(x)\right| \leq|W|^{1 / 2} . \\
& \forall x \in \mathbb{R}^{2}, \forall \lambda \in \mathbb{R}^{2},\left|F_{\lambda}(x)\right| \leq|W|^{1 / 2} .
\end{aligned}
$$

v) For $x \in \mathbb{R}^{2}$, we denote by $x^{+}$the only point in the orbit $W x$ which lies in $\overline{\mathfrak{a}^{+}}$. Then we have

$$
\forall x \in \mathbb{R}^{2}, G_{0}(x) \asymp \prod_{\substack{\alpha \in \mathcal{R}_{+} \\\langle\alpha, x\rangle \geq 0}}(1+\langle\alpha, x\rangle) e^{-\left\langle\rho, x^{+}\right\rangle} .
$$

vi) The function $F_{0}$ satisfies the estimate

$$
\forall x \in \overline{\mathfrak{a}_{+}}, F_{0}(x) \asymp e^{-\langle\rho, x\rangle} \prod_{\alpha \in \mathcal{R}_{+}}(1+\langle\alpha, x\rangle) .
$$

vii) Let $p$ and $q$ be polynomials of degree $m$ and $n$. Then there exists a positive constant $M$ such that for all $x \in \mathbb{R}^{2}$ and $\lambda \in \mathbb{C}^{2}$, we have

$$
\left|p\left(\frac{\partial}{\partial \lambda}\right) q\left(\frac{\partial}{\partial x}\right) G_{\lambda}(x)\right| \leq M(1+\|\lambda\|)^{n}(1+\|x\|)^{m} F_{0}(x) e^{\max _{w \in W} \operatorname{Im}\langle w \lambda, x\rangle} .
$$


viii) The function $G_{\lambda}, \lambda \in \mathbb{C}^{2}$, admits the following Laplace type representation

$$
\forall x \in \mathbb{R}^{2}, G_{\lambda}(x)=\int_{\mathbb{R}^{2}} e^{-i\langle\lambda, y\rangle} d \mu_{x}(y),
$$

where $\mu_{x}$ is the positive measure on $\mathbb{R}^{2}$ with support in $\Gamma=$ $\operatorname{conv}\{w x, w \in W\}$ (the convexe hull of the orbit of $x$ under $W$ ) given by the relation (4.33) of [11].

ix) From $(2.14),(2.23)$ we deduce that the function $F_{\lambda}, \lambda \in \mathbb{C}^{2}$, possesses the Laplace type representation

$$
\forall x \in \mathbb{R}^{2}, F_{\lambda}(x)=\int_{\mathbb{R}^{2}} e^{-i\langle\lambda, y\rangle} d \mu_{x}^{W}(y),
$$

where $\mu_{x}^{W}$ is the positive measure with support in $\Gamma$ given by

$$
\mu_{x}^{W}=\frac{1}{|W|} \sum_{w \in W} \mu_{w x}
$$

\section{The transmutation operators associated with the Chered- nik operators}

Notations. We denote by

- $\mathcal{E}\left(\mathbb{R}^{2}\right)$ the space of $C^{\infty}$-functions on $\mathbb{R}^{2}$. Its topology is defined by the seminorms

$$
q_{n, K}(\varphi)=\sup _{\substack{|\mu| \leq n \\ x \in K}}\left|D^{\mu} \varphi(x)\right|,
$$

where $K$ is a compact of $\mathbb{R}^{2}, n \in \mathbb{N}$, and

$$
D^{\mu}=\frac{\partial^{|\mu|}}{\partial^{\mu_{1}} x_{1} \partial^{\mu_{2}} x_{2}}, \mu=\left(\mu_{1}, \mu_{2}\right) \in \mathbb{N}^{2},|\mu|=\mu_{1}+\mu_{2} .
$$
have

- $\mathcal{D}\left(\mathbb{R}^{2}\right)$ the space of $C^{\infty}$-functions on $\mathbb{R}^{2}$ with compact support. We

$$
\mathcal{D}\left(\mathbb{R}^{2}\right)=\bigcup_{a>0} \mathcal{D}_{a}\left(\mathbb{R}^{2}\right)
$$

where $\mathcal{D}_{a}\left(\mathbb{R}^{2}\right)$ is the space of $C^{\infty}$-functions on $\mathbb{R}^{2}$ with support in the closed ball $B(0, a)$ of center 0 and radius $a$. The topology of $\mathcal{D}_{a}\left(\mathbb{R}^{2}\right)$ is defined by the semi-norms

$$
p_{n}(\psi)=\sup _{\substack{|\mu| \leq n \\ x \in B(0, a)}}\left|D^{\mu} \psi(x)\right|, \quad n \in \mathbb{N} .
$$


The space $\mathcal{D}\left(\mathbb{R}^{2}\right)$ is equipped with the inductive limit topology.

- $\mathcal{S}\left(\mathbb{R}^{2}\right)$ the classical Scwartz space on $\mathbb{R}^{2}$. Its topology is defined by the seminorms

$$
Q_{\ell, n}(f)=\sup _{\substack{|\mu| \leq n \\ x \in \mathbb{R}^{2}}}\left(1+\|x\|^{2}\right)^{\ell}\left|D^{\mu} f(x)\right|, \quad n, \ell \in \mathbb{N} .
$$

- $\mathcal{S}_{2}\left(\mathbb{R}^{2}\right)$ the generalized Schwartz space of $C^{\infty}$-functions on $\mathbb{R}^{2}$ such that for all $\ell, n \in \mathbb{N}$, we have

$$
P_{\ell, n}(f)=\sup _{\substack{|\mu| \leq n \\ x \in \mathbb{R}^{2}}}\left(1+\|x\|^{2}\right)^{\ell}\left(F_{0}(x)\right)^{-1}\left|D^{\mu} f(x)\right|<+\infty,
$$

where $F_{0}(x)$ is the Opdam-Cherednik kernel corresponding to the eigenvalue zero.

It is topologized by means of the seminorms $P_{\ell, n}, \ell, n \in \mathbb{N}$.

By using for $x \in \mathbb{R}^{2}$, the positive measure $\mu_{x}$ given by (2.23), we define the transmutation operator called also the trigonometric Dunkl intertwining operator $V_{k}$ on $\mathcal{E}\left(\mathbb{R}^{2}\right)$ by

$$
\forall x \in \mathbb{R}^{2}, V_{k}(g)(x)=\int_{\mathbb{R}^{2}} g(y) d \mu_{x}(y) .
$$

The operator $V_{k}$ is the unique linear topological isomorphism from $\mathcal{E}\left(\mathbb{R}^{2}\right)$ onto itself satisfying the transmutation relations

$$
\forall x \in \mathbb{R}^{2}, T_{j} V_{k}(g)(x)=V_{k}\left(\frac{\partial}{\partial y_{j}} g\right)(x), j=1,2,
$$

and the condition

$$
V_{k}(g)(0)=g(0) .
$$

The dual ${ }^{t} V_{k}$ of the operator $V_{k}$ is defined by the following duality relation

$$
\int_{\mathbb{R}^{2}}{ }^{t} V_{k}(f)(y) g(y) d y=\int_{\mathbb{R}^{2}} V_{k}(g)(x) f(x) \mathcal{A}_{k}(x) d x,
$$

with $f$ in $\mathcal{D}\left(\mathbb{R}^{2}\right)$ and $g$ in $\mathcal{E}\left(\mathbb{R}^{2}\right)$.

This operator is given by

$$
\forall y \in \mathbb{R}^{2},{ }^{t} V_{k}(f)(y)=\int_{\mathbb{R}^{2}} f(x) d \nu_{y}(x),
$$

where $\nu_{y}, y \in \mathbb{R}^{2}$, is the positive measure on $\mathbb{R}^{2}$ given by the relation (4.29) of [11], and verifying

$$
\nu_{y}(K)<+\infty \text {, for every compact } K \subset \mathbb{R}^{2} .
$$


The operator ${ }^{t} V_{k}$ is a linear topological isomorphism from

- $\mathcal{D}\left(\mathbb{R}^{2}\right)$ onto itself,

- $\mathcal{S}_{2}\left(\mathbb{R}^{2}\right)$ onto $\mathcal{S}\left(\mathbb{R}^{2}\right)$

satisfying the transmutation relations

$$
\forall y \in \mathbb{R}^{2},{ }^{t} V_{k}\left(\left(T_{j}+S_{j}\right) f\right)(y)=\frac{\partial}{\partial y_{j}}{ }^{t} V_{k}(f)(y), \quad j=1,2,
$$

where $S_{j}$ is the operator on $\mathcal{D}\left(\mathbb{R}^{2}\right)$ (resp. $\left.\mathcal{S}_{2}\left(\mathbb{R}^{2}\right)\right)$ given by

$$
\forall x \in \mathbb{R}^{2}, S_{j}(h)(x)=\sum_{\alpha \in \mathcal{R}_{+}} k(\alpha) \alpha^{j} h\left(r_{\alpha} x\right) .
$$

The operator ${ }^{t} V_{k}$ possesses also the following property : for all $f$ in $\mathcal{D}\left(\mathbb{R}^{2}\right)$ we have

$$
\operatorname{supp} f \subset B(0, a) \Leftrightarrow \operatorname{supp}^{t} V_{k}(f) \subset B(0, a),
$$

where $B(0, a)$ is the closed ball of center 0 and radius $a>0$.

REMARK 3.1. By using the measures $\mu_{x}^{W}, x \in \mathbb{R}^{2}$, and $\nu_{y}, y \in \mathbb{R}^{2}$ given by (2.25) and (3.5) we have defined and studied in [8] the trigonometric Dunkl intertwining operator $V_{k}^{W}$ on $\mathcal{E}\left(\mathbb{R}^{2}\right)^{W}$ (the subspace of functions of $\mathcal{E}\left(\mathbb{R}^{2}\right)$ which are $W$-invariant) and its dual ${ }^{t} V_{k}^{W}$ on $\mathcal{S}_{2}\left(\mathbb{R}^{2}\right)^{W}$ (the subspace of functions of $\mathcal{S}_{2}\left(\mathbb{R}^{2}\right)$ which are $W$-invariant), and we have given some properties of these operators.

\section{The hypergeometric Fourier transform associated with the Cherednik operators}

Notations.

For $a>0$ we denote by $P W\left(\mathbb{C}^{2}\right)_{a}$ the space of functions $h$ which are entire on $\mathbb{C}^{2}$ and satisfying

$$
\forall m \in \mathbb{N}, \mathfrak{s}_{m}(f)=\sup _{\lambda \in \mathbb{C}^{2}}\left(1+\|\lambda\|^{2}\right)^{m}|h(\lambda)| e^{-a\|\operatorname{Im}(\lambda)\|}<+\infty .
$$

Its topology is given by the seminorms $s_{m}, m \in \mathbb{N}$.

We consider the space $P W\left(\mathbb{C}^{2}\right)$ of entire functions on $\mathbb{C}^{2}$ which are rapidly decreasing and of exponential type. We have

$$
P W\left(\mathbb{C}^{2}\right)=\cup_{a>0} P W\left(\mathbb{C}^{2}\right)_{a}
$$


It is equipped with the inductive limit topology.

Definition 4.1. The hypergeometric Fourier transform $\mathcal{H}$ is defined for $f$ in $\mathcal{D}\left(\mathbb{R}^{2}\right)$ by

$$
\forall \lambda \in \mathbb{C}^{2}, \mathcal{H}(f)(\lambda)=\int_{\mathbb{R}^{2}} f(x) G_{\lambda}(x) \mathcal{A}_{k}(x) d x .
$$

Theorem 4.2. The transform $\mathcal{H}$ is a topological isomorphism from

i) $\mathcal{D}\left(\mathbb{R}^{2}\right)$ onto $P W\left(\mathbb{C}^{2}\right)$.

ii) $\mathcal{S}_{2}\left(\mathbb{R}^{2}\right)$ onto $\mathcal{S}\left(\mathbb{R}^{2}\right)$.

The inverse transform $\mathcal{H}^{-1}$ is given by

$$
\forall x \in \mathbb{R}^{2}, \mathcal{H}^{-1}(h)(x)=\int_{\mathbb{R}^{2}} h(\lambda) G_{\lambda}(-x) \mathcal{C}_{k}(\lambda) d \lambda,
$$

where

$$
\forall \lambda \in \mathbb{R}^{2}, \mathcal{C}_{k}(\lambda)=c\left|C_{k}(\lambda)\right|^{-2} \prod_{\alpha \in \mathcal{R}_{+}}\left(1+\frac{k(\alpha)}{i\langle\lambda, \breve{\alpha}\rangle}\right) .
$$

with $c$ a normalizing constant and

$$
\forall \lambda \in \mathbb{R}^{2},\left(C_{k}(\lambda)\right)^{-1}=\prod_{\alpha \in \mathcal{R}_{+}} \frac{\Gamma(i\langle\lambda, \breve{\alpha}\rangle+k(\alpha))}{\Gamma(i\langle\lambda, \breve{\alpha}\rangle)}
$$

We have

$$
\forall \lambda \in \mathbb{R}^{2},\left|C_{k}(\lambda)\right|^{2}=C_{k}(\lambda) C_{k}(-\lambda)=C_{k}(\lambda) \overline{C_{k}(\lambda)} .
$$

REMARK 4.3. The function $\mathcal{C}_{k}(\lambda)$ is continuous on $\mathbb{R}^{2}$ and satisfies the following estimate

$$
\forall \lambda \in \mathbb{R}^{2},\left|\mathcal{C}_{k}(\lambda)\right| \leq \text { const }\left(1+\|\lambda\|^{2}\right)^{b}
$$

for some $b>0$.

5. The hypergeometric translation operator and its dual and the hypergeometric convolution product associated with the Cherednik operators

5.1. The hypergeometric translation operator and its dual. The hypergeometric translation operator $\mathcal{T}_{x}, x \in \mathbb{R}^{2}$, (see [7]), is defined on $\mathcal{E}\left(\mathbb{R}^{2}\right)$ by

$$
\forall y \in \mathbb{R}^{2}, \mathcal{T}_{x}(f)(y)=\left(V_{k}\right)_{x}\left(V_{k}\right)_{y}\left[V_{k}^{-1}(f)(x+y)\right]
$$


We give the following some properties of the operator $\mathcal{T}_{x}, x \in \mathbb{R}^{2}$.

1. For all $x \in \mathbb{R}^{2}$, the operator $\mathcal{T}_{x}$ is continuous from $\mathcal{E}\left(\mathbb{R}^{2}\right)$ into itself.

2. For all $f$ in $\mathcal{E}\left(\mathbb{R}^{2}\right)$ and $x, y \in \mathbb{R}^{2}$, we have

$$
\mathcal{T}_{x}(f)(0)=f(x) \quad \text { and } \tau_{x}(f)(y)=\mathcal{T}_{y}(f)(x) .
$$

3. For all $x, y \in \mathbb{R}^{2}$ and $\lambda \in \mathbb{C}^{2}$, we have the product formula

$$
\mathcal{T}_{x}\left(G_{\lambda}\right)(y)=G_{\lambda}(x) G_{\lambda}(y),
$$

where $G_{\lambda}(x)$ is the Opdam-Cherednik kernel given by (2.13).

For each $x \in \mathbb{R}^{2}$, the dual of the hypergeometric translation operator $\mathcal{T}_{x}$ is the operator ${ }^{t} \mathcal{T}_{x}$ defined on $\mathcal{D}\left(\mathbb{R}^{2}\right)$ (resp. $\mathcal{S}_{2}\left(\mathbb{R}^{2}\right)$ ) (see [7]) by

$$
\forall y \in \mathbb{R}^{2},{ }^{t} \mathcal{T}_{x}(f)(y)=\left(V_{k}\right)_{x}\left({ }^{t} V_{k}^{-1}\right)_{y}\left[{ }^{t} V_{k}(f)(y-x)\right] .
$$

It satisfies the following properties.

1. For all $x \in \mathbb{R}^{2}$, the operator ${ }^{t} \mathcal{T}_{x}$ is continuous from

- $\mathcal{D}\left(\mathbb{R}^{2}\right)$ into itself.

- $\mathcal{S}_{2}\left(\mathbb{R}^{2}\right)$ into itself.

2. The operator ${ }^{t} \mathcal{T}_{x}, x \in \mathbb{R}^{2}$, is related to the operator $\mathcal{T}_{x}, x \in \mathbb{R}^{2}$, by the following two relations

i) For $g$ in $\mathcal{E}\left(\mathbb{R}^{2}\right)$ and $f$ in $\mathcal{D}\left(\mathbb{R}^{2}\right)\left(\right.$ resp. $\left.\mathcal{S}_{2}\left(\mathbb{R}^{2}\right)\right)$ we have

$$
\int_{\mathbb{R}^{2}} \mathcal{T}_{x}(g)(z) f(z) \mathcal{A}_{k}(z) d z=\int_{\mathbb{R}^{2}} g(y)^{t} \mathcal{T}_{x}(f)(y) \mathcal{A}_{k}(y) d y .
$$

ii) For $f$ in $\mathcal{D}\left(\mathbb{R}^{2}\right)$ (resp. $\left.\mathcal{S}_{2}\left(\mathbb{R}^{2}\right)\right)$ we have

$$
\forall x, y \in \mathbb{R}^{2},{ }^{t} \mathcal{T}_{x}(f)(y)=\mathcal{T}_{y}(\breve{f})(-x),
$$

where $\breve{f}$ is the function given by

$$
\forall x \in \mathbb{R}^{2}, \breve{f}(x)=f(-x) .
$$

3. For all $f$ in $\mathcal{D}\left(\mathbb{R}^{2}\right)$ (resp. $\mathcal{S}_{2}\left(\mathbb{R}^{2}\right)$ ) and $x \in \mathbb{R}^{2}$, we have

$$
\forall \lambda \in \mathbb{R}^{2}, \mathcal{H}\left({ }^{t} \mathcal{T}_{x}(f)\right)(\lambda)=G_{\lambda}(x) \mathcal{H}(f)(\lambda) .
$$

4. For all $f$ in $\mathcal{D}\left(\mathbb{R}^{2}\right)$ (resp. $\mathcal{S}_{2}\left(\mathbb{R}^{2}\right)$ ) and $x, y \in \mathbb{R}^{2}$, we have

$$
{ }^{t} \mathcal{T}_{x}(f)(y)=\int_{\mathbb{R}^{2}} G_{\lambda}(x) G_{\lambda}(-y) \mathcal{H}(f)(\lambda) \mathcal{C}_{k}(\lambda) d \lambda .
$$

5. For all $f$ in $\mathcal{D}\left(\mathbb{R}^{2}\right)$ (resp. $\mathcal{S}_{2}\left(\mathbb{R}^{2}\right)$ ) and $x, y \in \mathbb{R}^{2}$, we have

$$
{ }^{t} \mathcal{T}_{x}(f)(y)={ }^{t} \mathcal{T}_{-y}(f)(-x)
$$


6. For all $f$ in $\mathcal{D}\left(\mathbb{R}^{2}\right)$ with support in the closed ball $B(0, a)$ of center 0 and radius $a>0$, and $x \in \mathbb{R}^{2}$, we have

$$
\operatorname{supp}^{t} \mathcal{T}_{x}(f) \subset B(0, a+\|x\|) .
$$

5.2. The hypergeometric convolution product. By using the operator ${ }^{t} \mathcal{T}_{x}, x \in \mathbb{R}^{2}$, we define the hypergeometric convolution product $f *_{\mathcal{H}} g$ of the functions $f, g$ in $\mathcal{D}\left(\mathbb{R}^{2}\right)\left(\right.$ resp. $\mathcal{S}_{2}\left(\mathbb{R}^{2}\right)$ ) (see [7]) by

$$
\forall y \in \mathbb{R}^{2}, f *_{\mathcal{H}} g(y)=\int_{\mathbb{R}^{2}}{ }^{t} \mathcal{T}_{x}(f)(y) g(x) \mathcal{A}_{k}(x) d x .
$$

The hypergeometric convolution product $*_{\mathcal{H}}$ satisfies the following properties.

1. It is commutative and associative.

2. For all $f, g$ in $\mathcal{D}\left(\mathbb{R}^{2}\right)$ (resp. $\mathcal{S}_{2}\left(\mathbb{R}^{2}\right)$ ) the function $f * \mathcal{H} g$ belongs to $\mathcal{D}\left(\mathbb{R}^{2}\right)\left(\right.$ resp. $\mathcal{S}_{2}\left(\mathbb{R}^{2}\right)$ )

3. For all $f, g$ in $\mathcal{D}\left(\mathbb{R}^{2}\right)$ (resp. $\left.\mathcal{S}_{2}\left(\mathbb{R}^{2}\right)\right)$, we have

$$
\forall \lambda \in \mathbb{R}^{2}, \mathcal{H}\left(f *_{\mathcal{H}} g\right)(\lambda)=\mathcal{H}(f)(\lambda) . \mathcal{H}(g)(\lambda) .
$$

4. For all $f, g$ in $\mathcal{D}\left(\mathbb{R}^{2}\right)$ (resp. $\mathcal{S}_{2}\left(\mathbb{R}^{2}\right)$ ), we have

$$
{ }^{t} V_{k}(f * \mathcal{H} g)={ }^{t} V_{k}(f) *{ }^{t} V_{k}(g),
$$

where $*$ is the classical convolution product on $\mathbb{R}^{2}$.

\section{The heat kernel associated with the Cherednik operators}

Definition 6.1. Let $t>0$. The heat kernel $p_{t}(x, y)$ associated with the Cherednik operators, is defined for $x, y \in \mathbb{R}^{2}$, by

$$
p_{t}(x, y)=\int_{\mathbb{R}^{2}} e^{-t\left(\|\lambda\|^{2}+\|\rho\|^{2}\right)} G_{\lambda}(x) G_{\lambda}(-y) \mathcal{C}_{k}(\lambda) d \lambda .
$$

Notations. We denote by

- $H_{k}$ the heat operator associated with the Cherednik operators given by

$$
H_{k}=\mathcal{L}_{k}-\frac{\partial}{\partial t}-\|\rho\|^{2}
$$

where $\mathcal{L}_{k}$ is the Heckman-Opdam Laplacian defined for all $f$ of class $C^{2}$ on $\mathbb{R}^{2}$ by

$$
\mathcal{L}_{k} f=\left(T_{1}+T_{2}\right) f
$$


It has the following form : For all $x \in \mathbb{R}_{\text {reg }}^{2}$,

$$
\begin{aligned}
\mathcal{L}_{k} f(x) & =\Delta f(x)+\sum_{\alpha \in \mathcal{R}_{+}} k(\alpha) \operatorname{coth}\left(\frac{\langle\alpha, x\rangle}{2}\right)\langle\nabla f(x), \alpha\rangle \\
& +\sum_{\alpha \in \mathcal{R}_{+}} \frac{k(\alpha)\|\alpha\|^{2}}{4 \sinh ^{2} \frac{\langle\alpha, x\rangle}{2}}\left\{f\left(r_{\alpha} x\right)-f(x)\right\}+\|\rho\|^{2} f(x) .
\end{aligned}
$$

where $\Delta$ and $\nabla$ are respectively the Laplacian and the gradient on $\mathbb{R}^{2}$.

- $E_{t}, t>0$, the fundamental solution of the operator $H_{k}$ given by

$$
\forall x \in \mathbb{R}^{2}, E_{t}(x)=p_{t}(x, 0) .
$$

\section{PROPOSITION 6.2.}

i) For all $t>0$, the function $E_{t}$ belongs to $\mathcal{S}_{2}\left(\mathbb{R}^{2}\right)$.

ii) For all $t>0$ and $x \in \mathbb{R}^{2}$, we have

$$
E_{t}(x) \asymp t^{-\gamma-1}\left[\prod_{\alpha \in \mathcal{R}_{+}} M_{\alpha}(t, x)\right] e^{-t\|\rho\|^{2}-\left\langle\rho, x^{+}\right\rangle-\frac{\|x\|^{2}}{4 t}},
$$

where $x^{+}$denotes the unique conjugate in $\overline{\mathfrak{a}_{+}}$,

$$
\gamma=\sum_{\alpha \in \mathcal{R}_{+}} k(\alpha)
$$

and

$$
M_{\alpha}(t, x)=2^{-\gamma-1}\left[\left(1+\left|\left\langle\alpha_{i}, x\right\rangle\right|\right)(1+2 t+|\langle\alpha, x\rangle|)^{k(\alpha)-1}\right]
$$

iii) For all $t>0$, we have

$$
\forall \lambda \in \mathbb{R}^{2}, \mathcal{H}\left(E_{t}\right)(\lambda)=e^{-t\left(\|\lambda\|^{2}+\|\rho\|^{2}\right)} .
$$

iv) The function $(x, t) \rightarrow E_{t}(x)$ is strictly positive on $\left.\mathbb{R}^{2} \times\right] 0,+\infty[$.

v) For all $t>0$, we have

$$
\int_{\mathbb{R}^{2}} E_{t}(x) \mathcal{A}_{k}(x) d x=1 .
$$

vi) We have

$$
\left.H_{h} E_{t}(x)=0, \quad \text { on } \mathbb{R}^{2} \times\right] 0,+\infty[.
$$

Proposition 6.3.

i) For all $t>0$ and $x \in \mathbb{R}^{2}$, the function $y \rightarrow p_{t}(x, y)$ belongs to $\mathcal{S}_{2}\left(\mathbb{R}^{2}\right)$.

ii) For all $t>0$ and $x, y \in \mathbb{R}^{2}$, we have

$$
p_{t}(x, y)={ }^{t} \mathcal{T}_{x}\left(E_{t}\right)(y) .
$$

iii) The function $p_{t}(x, y)$ is strictly positive on $\left.\mathbb{R}^{2} \times \mathbb{R}^{2} \times\right] 0,+\infty[$. 
iv) For all $t>0$ and $x \in \mathbb{R}^{2}$, we have

$$
\int_{\mathbb{R}^{2}} p_{t}(x, y) \mathcal{A}_{k}(y) d y=1 .
$$

v) For all $y \in \mathbb{R}^{d}$, the function $(x, t) \rightarrow p_{t}(x, y)$ satisfies

$$
\left.H_{k} p_{t}(x, y)=0, \text { on } \mathbb{R}^{2} \times\right] 0,+\infty[\text {. }
$$

Definition 6.4. The heat semigroup $\left\{P_{t}\right\}_{t \geq 0}$ associated with the Cherednik operators, is defined for $f$ in $\mathcal{S}_{2}\left(\mathbb{R}^{2}\right)$ by

$$
\forall x \in \mathbb{R}^{2}, P_{t} f(x)= \begin{cases}\int_{\mathbb{R}^{2}} p_{t}(x, y) f(y) \mathcal{A}_{k}(y) d y & \text { if } t>0, \\ f(x) & \text { if } t=0 .\end{cases}
$$

(See $[5])$.

REMARK 6.5. The function $P_{t} f(x)$ can also be written in the following form

$$
\forall x \in \mathbb{R}^{2}, P_{t} f(x)=E_{t} * \mathcal{H} \breve{f}(-x),
$$

where $*_{\mathcal{H}}$ is the hypergeometric convolution product given by the relation (5.11), and $\vec{f}$ is the function defined by

$$
\forall x \in \mathbb{R}^{2}, \breve{f}(x)=f(x) .
$$

We consider the Cauchy problem : Given a continuous bounded function on $\mathbb{R}^{2}$. Find a function $u(x, t)$ of class $C^{2}$ on $\left.\mathbb{R}^{2} \times\right] 0,+\infty[$, such that

$$
\begin{cases}H_{k} u(x, t) & \left.=0, \text { on } \mathbb{R}^{2} \times\right] 0,+\infty[ \\ u(x, 0) & =f(x)\end{cases}
$$

Proposition 6.6.

i) $\left\{P_{t}\right\}_{t \geq 0}$ is a strongly continuous semigroup on $\mathcal{S}_{2}\left(\mathbb{R}^{2}\right)$.

ii) Let $f$ be a continuous bounded function on $\mathbb{R}^{2}$. Then the function $u(x, t)=P_{t} f(x)$ solves the Cauchy problem (6.17).

(See [5]).

7. Positivity of the function ${ }^{t} \mathcal{T}_{x}\left(p_{t}(u,).(y)\right.$

Proposition 7.1. Let $t>0$ and $x \in \mathbb{R}^{2}$. We have $\forall u, y \in \mathbb{R}^{2},{ }^{t} \mathcal{T}_{x}\left(p_{t}(u,).\right)(y)=$

$$
\int_{\mathbb{R}^{2}} e^{-t\left(\|\lambda\|^{2}+\|\rho\|^{2}\right)} G_{\lambda}(x) G_{\lambda}(u) G_{\lambda}(-y) \mathcal{C}_{k}(\lambda) d \lambda .
$$


Proof. From Proposition 6.1 i), for all $u \in \mathbb{R}^{2}$, the function $z \rightarrow$ $p_{t}(u, z)$ belongs to $\mathcal{S}_{2}\left(\mathbb{R}^{2}\right)$. Then by using (5.8) we obtain

$$
\forall u, z \in \mathbb{R}^{2},{ }^{t} \mathcal{T}_{x}\left(p_{t}(u, .)\right)(y)=\int_{\mathbb{R}^{2}} G_{\lambda}(x) G_{\lambda}(-y) \mathcal{H}\left(p_{t}(u, .)\right)(\lambda) \mathcal{C}_{k}(\lambda) d \lambda .
$$

On the other hand from (6.12), (5.7), we have

$$
\forall u, \lambda \in \mathbb{R}^{2}, \mathcal{H}\left(p_{t}(u, .)\right)(\lambda)=\mathcal{H}\left({ }^{t} \mathcal{T}_{u}\left(E_{t}\right)\right)(\lambda)=G_{\lambda}(u) \mathcal{H}\left(E_{t}\right)(\lambda),
$$

thus from (6.9) we obtain

$$
\forall u, \lambda \in \mathbb{R}^{2}, \mathcal{H}\left(p_{t}(u, .)\right)(\lambda)=e^{-t\left(\|\lambda\|^{2}+\|\rho\|^{2}\right)} G_{\lambda}(u) .
$$

We deduce (7.1) from (7.2), (7.3).

Corollary 7.2. Let $t>0$ and $x \in \mathbb{R}^{2}$. We have

$$
\forall u, y \in \mathbb{R}^{2},{ }^{t} \mathcal{T}_{x}\left(p_{t}(u, .)\right)(y)=e^{-t\|\rho\|^{2}} \int_{\mathbb{R}^{2}} U_{t, x}(-y, z) d \breve{\mu}_{u}(z),
$$

where $\mu_{u}$ is the measure given by (2.23) and $\breve{\mu}_{u}$ is the measure on $\mathbb{R}^{2}$ defined by

$$
\int_{\mathbb{R}^{2}} f(z) d \breve{\mu}_{u}(z)=\int_{\mathbb{R}^{2}} f(-z) d \mu_{u}(z), \quad f \in \mathcal{E}\left(\mathbb{R}^{2}\right),
$$

and

$$
U_{t, x}(y, z)=\int_{\mathbb{R}^{2}} e^{-t\|\lambda\|^{2}} G_{\lambda}(x) G_{\lambda}(y) e^{i\langle\lambda, z\rangle} \mathcal{C}_{k}(\lambda) d \lambda .
$$

Proof. We deduce (7.4) from (7.1), (2.18), (2.23), the positivity of the measure $\breve{\mu}_{x}$ and Fubini's theorem.

In this section we prove first that for $t>0$ and $x \in \mathbb{R}^{2}$, the function $(y, z) \rightarrow U_{t, x}(y, z)$ is positive on $\mathbb{R}^{2} \times \mathbb{R}^{2}$, and next we deduce the positivity of the function ${ }^{t} \mathcal{T}_{x}\left(p_{t}(u,).\right)(y)$.

Proposition 7.3. Let $t>0$ and $x \in \mathbb{R}^{2}$. The function $U_{t, x}(y, z)$ is of class $C^{\infty}$ on $\mathbb{R}^{2} \times \mathbb{R}^{2}$ with respect to the variables $y$ and $z$ and satisfies the equation

$$
\forall y, z \in \mathbb{R}^{2},\left(T_{j}+\frac{\partial}{\partial z_{j}}\right) U_{t, x}(y, z)=0, \quad j=1,2 .
$$

Proof. We obtain the results by derivation under the integral sign with respect to the variables $y_{j}, z_{j}, j=1,2$, in the relation (7.6) by using $(2.22),(2.19)$, and by applying the relation (2.13). 
Proposition 7.4.

i) Let $t>0$ and $x \in \mathbb{R}^{2}$. There exists a positive function $M_{0}(t)$ such that

$\forall y, z \in \mathbb{R}^{2},\left|U_{t, x}(y, z)\right| \leq M_{0}(t) \prod_{\alpha \in \mathcal{R}_{+}}(1+|\langle\alpha, y\rangle|) e^{-\left\langle\rho, y^{+}\right\rangle}$,

where $y^{+}$is the only point in the orbit $W y$ which lies in $\overline{\mathfrak{a}^{+}}$.

ii) Let $t>0$ and $x \in \mathbb{R}^{2}$. We have

$$
\forall y \in \mathbb{R}^{2}, \lim _{\|z\| \rightarrow+\infty} U_{t, x}(y, z)=0 .
$$

iii) Let $t>0$ and $x \in \mathbb{R}^{2}$. The function $(y, z) \rightarrow U_{t, x}(y, z)$ is bounded on $\mathbb{R}^{2} \times \mathbb{R}^{2}$ and we have

$$
\lim _{\|(y, z)\| \rightarrow+\infty} U_{t, x}(y, z)=0 .
$$

Proof.

i) We deduce (7.8) from (7.6), (2.17), (2.20).

ii) By using (7.6) and the fact that from (2.18) the function $e^{-t\|\lambda\|^{2}} G_{\lambda}(x) G_{\lambda}(y)$ is for all $x, y \in \mathbb{R}^{2}$, integrable with respect to the Lebesgue measure on $\mathbb{R}^{2}$, we deduce (7.9) from Riemann-Lebesgue Lemma.

iii) - The relations (7.6), (2.18) imply that the function $(y, z) \rightarrow U_{t, x}(y, z)$ is bounded on $\mathbb{R}^{2} \times \mathbb{R}^{2}$.

- We deduce (7.10) from (7.8), (7.9).

\section{Proposition 7.5.}

i) Let $t>0$ and $x \in \mathbb{R}^{2}$. For all $y, z \in \mathbb{R}^{2}$ the function $U_{t, x}(y, z)$ is real.

ii) Let $t>0$ and $x \in \mathbb{R}^{2}$. The function $U_{t, x}(y, z)$ is strictly positive on the set

$$
Y=\left\{(y, z) \in \mathbb{R}^{2} \times \mathbb{R}^{2} ; y \in \mathbb{R}^{2}, z=0\right\} .
$$

Proof.

i) We obtain the result from the relations (7.6), (2.16), (4.3), (4.5) and a change of variables.

ii) By using the relation

$$
\forall \lambda \in \mathbb{R}^{2}, G_{\lambda}(0)=1,
$$


we deduce from (7.6), (7.4), (3.1), (3.3), (6.1) and Proposition 6.2 iii), that

$$
\forall y \in \mathbb{R}^{2}, U_{t, x}(y, 0)=e^{-t\|\rho\|^{2}} p_{t}(x, y)>0 .
$$

Proposition 7.6. Let $t>0$ and $x \in \mathbb{R}^{2}$. For all $\alpha \in \mathcal{R}_{+}$, and $y, z \in \mathbb{R}^{2}$, we have

$$
\begin{aligned}
U_{t, x}\left(r_{\alpha_{i}} y, z\right)-U_{t, x}(y, z) & =-\langle\breve{\alpha}, y\rangle\left\langle\nabla U_{t, x}(y, z), \alpha\right\rangle \\
& +\frac{1}{2}(\langle\breve{\alpha}, y\rangle)^{2} \alpha^{t} D^{2} U_{t, x}(\xi, z) \alpha,
\end{aligned}
$$

with some $\xi$ on the line segment between $y$ and $r_{\alpha} y$.

Proof. We obtain (7.11) from the relation (2.5) and Taylor's formula.

Proposition 7.7. The Weyl chambers attached to the root system of type $B_{2}$ are the following

$$
\begin{gathered}
\left\{\begin{array}{l}
\mathfrak{a}^{+}=\left\{x \in \mathbb{R}^{2} ;\left\langle\alpha_{i}, x\right\rangle>0, i=1,2,3,4\right\} \\
\mathfrak{a}^{-}=-\mathfrak{a}^{+}
\end{array}\right. \\
\left\{\begin{array}{l}
\mathfrak{a}_{1}^{+}=\left\{x \in \mathbb{R}^{2} ;\left\langle\alpha_{i}, x\right\rangle>0, i=1,3,4 ;\left\langle\alpha_{2}, x\right\rangle<0\right\} \\
\mathfrak{a}_{1}^{-}=-\mathfrak{a}_{1}^{+}
\end{array}\right. \\
\left\{\begin{array}{l}
\mathfrak{a}_{2}^{+}=\left\{x \in \mathbb{R}^{2} ;\left\langle\alpha_{i}, x\right\rangle>0, i=1,2,4 ;\left\langle\alpha_{3}, x\right\rangle<0\right\} \\
\mathfrak{a}_{3}^{-}=-\mathfrak{a}_{2}^{+}
\end{array}\right. \\
\left\{\begin{array}{l}
\mathfrak{a}_{3}^{+}=\left\{x \in \mathbb{R}^{2} ;\left\langle\alpha_{i}, x\right\rangle>0, i=2,4 ;\left\langle\alpha_{i}, x\right\rangle<0, i=1,3\right\} \\
\mathfrak{a}_{3}^{-}=-\mathfrak{a}_{3}^{+}
\end{array}\right.
\end{gathered}
$$

Proof. We determine the Weyl chambers corresponding to the four roots of $\mathcal{R}_{+}$, and next by applying the relations

$$
\left\{\begin{array}{l}
\alpha_{1}-\alpha_{2}=\alpha_{3} \\
\alpha_{1}+\alpha_{2}=\alpha_{4}
\end{array}\right.
$$

we obtain the Weyl chambers (7.12), (7.13), (7.14), (7.15), the others are empty.

Notations. We denote by $C_{\ell}, \ell=1,2,3,4$, the Weyl chambers $\mathfrak{a}^{+}, \mathfrak{a}_{1}^{+}, \mathfrak{a}_{2}^{+}, \mathfrak{a}_{3}^{+}$and by $C_{\ell}, \ell=5,6,7,8$, the Weyl chambers $\mathfrak{a}^{-}, \mathfrak{a}_{1}^{-}, \mathfrak{a}_{2}^{-}, \mathfrak{a}_{3}^{-}$. Then we have

$$
\mathbb{R}^{2}=\bigcup_{\ell=1}^{8} \bar{C}_{\ell},
$$

where $\bar{C}_{\ell}$ is the closure of $C_{\ell}$. 
TheOREM 7.8. For all $t>0$, and $x \in \mathbb{R}^{2}$ we have

$$
\forall y, z \in \mathbb{R}^{2}, U_{t, x}(y, z) \geq 0 .
$$

Proof. The proof is made up in two steps.

In the first step we obtain some results concerning the positivity of the function $U_{t, x}(y, z)$ given by $(7.6)$ on each of the step $\overline{C_{\ell}} \times \mathbb{R}^{2}, \ell=1,2, \ldots, 8$.

In the second step we use the fact that $\mathbb{R}^{2} \times \mathbb{R}^{2}=\left(\cup_{\ell=1}^{8} \overline{C_{\ell}}\right) \times \mathbb{R}^{2}$ and the result of the first set to deduce the positivity of the function $U_{t, x}(y, z)$ on $\mathbb{R}^{2} \times \mathbb{R}^{2}$.

\section{ST STEP}

We consider the set $Y_{\ell}$ defined by

$$
Y_{\ell}=\left\{(y, z) \in \mathbb{R}^{2} \times \mathbb{R}^{2} ; \quad y \in \overline{C_{\ell}}, z \in \mathbb{R}^{2}\right\} .
$$

We denote by

$$
\mathcal{V}_{t, x}^{\ell}(y, z)=U_{t, x}(y, z) 1_{Y_{\ell}}(y, z)
$$

where $1_{Y_{\ell}}$ is the characteristic function of the set $Y_{\ell}$. From Proposition 7.5 ii) the function $\mathcal{V}_{t, x}^{\ell}(y, z)$ is strictly positive on the set $Y$. We shall prove that it is positive on the set $Y_{\ell} \backslash Y$. If not we suppose by using Proposition $7.5 \mathrm{i}$ ) and Proposition 7.4 iii) that it attains a strictly negative absolute minimum at $\left(y^{\ell}, z^{\ell}\right) \in Y_{\ell} \backslash Y$ i.e.

$$
\mathcal{V}_{t, x}^{\ell}\left(y^{\ell}, z^{\ell}\right)=\inf _{(y, z) \in Y_{\ell}} \mathcal{V}_{t, x}^{\ell}(y, z)<0
$$

There are two possibilities: The point $\left(y^{\ell}, z^{\ell}\right)$ is in the open subset $\left(Y_{\ell} \backslash Y\right)^{0}$ of the set $Y_{\ell} \backslash Y$, or in the set

$$
Y_{\ell}^{0}=\left\{(y, z) \in \mathbb{R}^{2} \times \mathbb{R}^{2} ; \quad y \in \partial \overline{C_{\ell}}, z \in \mathbb{R}^{2}\right\} .
$$

We suppose that $\left(y^{\ell}, z^{\ell}\right) \in\left(Y_{\ell} \backslash Y\right)^{0}$. As the point $\left(y^{\ell}, z^{\ell}\right)$ is an absolute minimum, then we have

$$
\frac{\partial}{\partial y_{j}} \mathcal{V}_{t, x}^{\ell}\left(y^{\ell}, z^{\ell}\right)=\frac{\partial}{\partial z_{j}} \mathcal{V}_{t, x}^{\ell}\left(y^{\ell}, z^{\ell}\right)=0, \quad j=1,2 .
$$

By using the fact that

$$
\forall \alpha \in \mathcal{R}_{+},\left(r_{\alpha} y^{\ell}, z^{\ell}\right) \notin Y_{\ell},
$$


and by applying the relations (7.7), (2.11), (2.12), (7.19), we get

$$
\begin{aligned}
&\left\{k_{1}\left(\frac{1}{1-e^{-\left\langle\alpha_{1}, x^{\ell}\right\rangle}}-\frac{1}{2}\right)+\right. \\
&\left.k_{2}\left[\left(\frac{1}{1-e^{-\left\langle\alpha_{3}, x^{\ell}\right\rangle}}-\frac{1}{2}\right)+\left(\frac{1}{1-e^{-\left\langle\alpha_{4}, x^{\ell}\right\rangle}}-\frac{1}{2}\right)\right]\right\} \mathcal{V}_{s}^{\ell}\left(x^{\ell}, u^{\ell}\right)=0, \\
&\{ k_{1}\left(\frac{1}{1-e^{-\left\langle\alpha_{2}, x^{\ell}\right\rangle}}-\frac{1}{2}\right)+ \\
&\left.k_{2}\left[-\left(\frac{1}{1-e^{-\left\langle\alpha_{3}, x^{\ell}\right\rangle}}-\frac{1}{2}\right)+\left(\frac{1}{1-e^{-\left\langle\alpha_{4}, x^{\ell}\right\rangle}}-\frac{1}{2}\right)\right]\right\} \mathcal{V}_{s}^{\ell}\left(x^{\ell}, u^{\ell}\right)=0,
\end{aligned}
$$

Using the fact that from (7.17) the function $\mathcal{V}_{s}^{\ell}\left(x^{\ell}, u^{\ell}\right)$ is different from zero and that $k_{2}>0$, the equations (7.20), (7.21) can also be written in the form

$$
\begin{aligned}
& \frac{k_{1}}{k_{2}} X_{1}^{\ell}+X_{3}^{\ell}+X_{4}^{\ell}=0 \\
& \frac{k_{1}}{k_{2}} X_{2}^{\ell}-X_{3}^{\ell}+X_{4}^{\ell}=0
\end{aligned}
$$

with

$$
X_{i}^{\ell}=\frac{1+e^{-\left\langle\alpha_{i}, x^{\ell}\right\rangle}}{1-e^{-\left\langle\alpha_{i}, x^{\ell}\right\rangle}}, i=1,2,3,4 .
$$

Then the $X_{i}^{\ell}, i=1,2,3,4$, are solutions of the system of linear equations $(S)$ on $\mathbb{R}^{4}$ :

$$
\text { (S) }\left\{\begin{array}{l}
\frac{k_{1}}{k_{2}} X_{1}+X_{3}+X_{4}=0, \\
\frac{k_{1}}{k_{2}} X_{2}-X_{3}+X_{4}=0 .
\end{array}\right.
$$

On the other hand from (7.24) we obtain

$$
e^{-\left\langle\alpha_{i}, x^{\ell}\right\rangle}=\frac{X_{i}^{\ell}-1}{X_{i}^{\ell}+1}, i=1,2,3,4
$$

We consider the function $f$ defined on $\mathbb{R} \backslash\{-1\}$ by

$$
f(y)=\frac{y-1}{y+1}
$$

we have

$$
\begin{gathered}
f(y) \leq 0 \Leftrightarrow y \in]-1,1], \\
0<f(y)<1 \Leftrightarrow y \in] 1,+\infty[, \\
f(y)>1 \Leftrightarrow y \in]-\infty,-1[
\end{gathered}
$$


From the relation $(7.26)$, we have

$$
e^{-\left\langle\alpha_{i}, x^{\ell}\right\rangle}=f\left(X_{i}^{\ell}\right), \quad i=1,2,3,4 .
$$

As the first member of (7.30) is strictly positive, then from (7.27) the $X_{i}^{\ell}, i=1,2,3,4$, are not in the interval ] - 1, 1[. They are in the interval ]$-\infty,-1[\cup] 1,+\infty[$. We consider two cases

1ST CASE

1. If $x^{\ell} \in C_{\ell}, \ell=1,2$.

From the relations (7.12), (7.13) we have $\left\langle\alpha_{i}, x^{\ell}\right\rangle>0$ for $i=1,3,4$.

Then by using (7.30), (7.28) we obtain

$$
\left.X_{i}^{\ell} \in\right] 1,+\infty[, \quad i=1,3,4 .
$$

By applying (7.31) we get

$$
\frac{k_{1}}{k_{2}} X_{1}^{\ell}+X_{3}^{\ell}+X_{4}^{\ell}>\frac{k_{1}}{k_{2}}+2>0 .
$$

Thus from (7.22) we obtain an absurdity, and then the $X_{i}^{\ell}, i=$ $1,2,3,4$, are not solutions of the system $(S)$ given by $(7.25)$.

2. If $x^{\ell} \in C_{\ell}, \ell=3,4$.

From the relation (7.14),(7.15) we have $\left\langle\alpha_{i}, x^{\ell}\right\rangle>0$ for $i=2,4$, and $\left\langle\alpha_{3}, x^{\ell}\right\rangle<0$. Then by using (7.30), (7.28), (7.29), we obtain

$$
\left.X_{i}^{\ell} \in\right] 1,+\infty\left[, i=2,4 \text {, and } X_{3}^{\ell} \in\right]-\infty,-1[.
$$

By applying (7.32) we get

$$
\frac{k_{1}}{k_{2}} X_{2}^{\ell}-X_{3}^{\ell}+X_{4}^{\ell}>\frac{k_{1}}{k_{2}}+2>0 .
$$

Thus from (7.23) we obtain an absurdity, and then the $X_{i}^{\ell}, i=$ $1,2,3,4$, are not solution of the system $(S)$ given by (7.25).

2ND CASE : If $x^{\ell} \in C_{\ell}, \ell=5,6,7,8$.

The same proof as for the first case shows that when $x^{\ell} \in C_{\ell}, \ell=$ $5,6,7,8$, we obtain an absurdity, and then the $X_{i}^{\ell}, i=1,2,3,4$, are not solutions of the system $(S)$ given by (7.25).

From the first and second cases we deduce that our supposition that the function $\mathcal{V}_{t, x}^{\ell}(y, z)$ attains a strictly negative absolute minimum at $\left(y^{\ell}, z^{\ell}\right)$ in $\left(Y_{\ell} \backslash Y\right)^{0}$ is absurd. Then the point $\left(y^{\ell}, z^{\ell}\right)$ does not belong to $\left(Y_{\ell} \backslash Y\right)^{0}$, and it is in the set $Y_{\ell}^{0}$. 


\section{ND STEP}

From Proposition 7.5 ii) the function $U_{t, x}(y, z)$ is strictly positive on the set $Y$.

We shall prove that the function $U_{t, x}(y, z)$ is positive on the set $\mathbb{R}^{2} \times$ $\mathbb{R}^{2} \backslash Y$. If not we suppose by using Proposition 7.5.i) and Proposition 7.4 iii) that it attains a strictly negative absolute minimum at $\left(y_{0}, z_{0}\right) \in$ $\mathbb{R}^{2} \times \mathbb{R}^{2} \backslash Y$.

From the first step and the relation $\mathbb{R}^{2}=\cup_{\ell=1}^{8} \bar{C}_{\ell}$, the point $\left(y_{0}, z_{0}\right)$ is in the set

$$
Y^{0}=\bigcup_{\ell=1}^{8} Y_{\ell}^{0},
$$

with $Y_{\ell}^{0}$ given by (7.18). We have

$$
Y^{0}=\left\{(y, z) \in \mathbb{R}^{2} \times \mathbb{R}^{2} ; \forall \alpha \in \mathcal{R}_{+},\langle\alpha, y\rangle=0, z \in \mathbb{R}^{2}\right\},
$$

then

$$
\forall \alpha \in \mathcal{R}_{+},\left\langle\alpha, y_{0}\right\rangle=0 .
$$

We shall prove in the following that the point $\left(y_{0}, z_{0}\right)$ is not in the set $Y^{0}$.

As the point $\left(y_{0}, z_{0}\right)$ is a strictly negative absolute minimum, then we have the following relations

$$
U_{t, x}\left(y_{0}, z_{0}\right)=\inf _{(y, z) \in \mathbb{R}^{2} \times \mathbb{R}^{2}} U_{t, x}(y, z)<0,
$$

and

$$
\frac{\partial}{\partial y_{1}} U_{t, x}\left(y_{0}, z_{0}\right)=\frac{\partial}{\partial z_{1}} U_{t, x}\left(y_{0}, z_{0}\right)=0
$$

We write the relations $(7.6),(7.7),(2.11)$ for $y, z_{0}$, and we get

$$
\begin{gathered}
\frac{\partial}{\partial y_{1}} U_{t, x}\left(y, z_{0}\right)+\frac{\partial}{\partial z_{1}} U_{t, x}\left(y, z_{0}\right)+k_{1} \frac{U_{t, x}\left(y, z_{0}\right)-U_{t, x}\left(r_{\alpha_{1}} y, z_{0}\right)}{1-e^{-\left\langle\alpha_{1}, x\right\rangle}}+ \\
k_{2}\left[\frac{U_{t, x}\left(y, z_{0}\right)-U_{t, x}\left(r_{\alpha_{3}} y, z_{0}\right)}{1-e^{-\left\langle\alpha_{3}, x\right\rangle}}+\frac{U_{t, x}\left(y, z_{0}\right)-U_{t, x}\left(r_{\alpha_{4}} y, z_{0}\right)}{1-e^{-\left\langle\alpha_{4}, x\right\rangle}}\right] \\
=\left(\frac{1}{2} k_{1}+k_{2}\right) U_{t, x}\left(y, z_{0}\right) .
\end{gathered}
$$

Then by passing to the limit in (7.35), when $\langle\alpha, y\rangle$, for all $\alpha \in \mathcal{R}_{+}$, goes to $\left\langle\alpha, y_{0}\right\rangle=0$, and by using Proposition 7.6 and the relation (7.34), we obtain

$$
\left(\frac{1}{2} k_{1}+k_{2}\right) U_{t, x}\left(y_{0}, z_{0}\right)=0 .
$$


As $k_{1}>0$, and $k_{2}>0$ then

$$
U_{t, x}\left(y_{0}, z_{0}\right)=0 .
$$

Thus (7.33) and (7.36) imply a contradiction, and the point $\left(y_{0}, z_{0}\right)$ is not in the set $Y^{0}$.

Then the function $U_{t, x}(y, z)$ is positive on the set $\mathbb{R}^{2} \times \mathbb{R}^{2} \backslash Y$. We deduce the relation (7.16) from this result and the fact that the function $U_{t, x}(y, z)$ is positive on the set $Y$.

Theorem 7.9. Let $t>0$ and $x \in \mathbb{R}^{2}$. Then we have

$$
\forall, u, y \in \mathbb{R}^{2},{ }^{t} \mathcal{T}_{x}\left(p_{t}(u, .)\right)(y) \geq 0 .
$$

Proof. We deduce (7.37) from the relation (7.4), the positivity of the measure $\breve{\mu}_{x}$ given by (7.5) and Theorem 7.8.

\section{Positivity of the hypergeometric translation operator and of its dual}

TheOREM 8.1. For all positive function $g$ in $\mathcal{E}\left(\mathbb{R}^{2}\right)$, we have

$$
\forall x, u \in \mathbb{R}^{2}, \mathcal{T}_{x}(g)(u) \geq 0 .
$$

Proof. I

- For $x=0$

From the relation (5.2) we have

$$
\mathcal{T}_{0}(g)(u)=g(u) \geq 0 .
$$

- For $x \in \mathbb{R}^{2} \backslash\{0\}$

We consider tow cases.

1ST CASE : We suppose that the function $g$ belongs to $\mathcal{D}\left(\mathbb{R}^{2}\right)$.

Let $t>0$ and $u \in \mathbb{R}^{2}$. From Proposition 6.2 i) the function $z \rightarrow p_{t}(u, z)$ belongs to $\mathcal{S}_{2}\left(\mathbb{R}^{2}\right)$. On the other hand by using the properties of the operators $\mathcal{T}_{x}$ and ${ }^{t} \mathcal{T}_{x}$ and the relations (5.6), (5.5), we obtain

$$
\int_{\mathbb{R}^{2}} \mathcal{T}_{x}(g)(z) p_{t}(u, z) \mathcal{A}_{k}(z) d z=\int_{\mathbb{R}^{2}} g(y)^{t} \mathcal{T}_{x}\left(p_{t}(u, .)\right)(y) \mathcal{A}_{k}(y) d y .
$$

As from (7.37) the second member of this relation is positive, then we have

$$
\int_{\mathbb{R}^{2}} \mathcal{T}_{x}(g)(z) p_{t}(u, z) \mathcal{A}_{k}(z) d z \geq 0
$$


By using (6.15) this relation can also be written in the form

$$
P_{t}\left(\mathcal{T}_{x}(g)\right)(u) \geq 0
$$

As the function $\mathcal{T}_{x}(g)$ belongs to $\mathcal{D}\left(\mathbb{R}^{2}\right)$, then from Proposition 6.5 ii) we obtain

$$
\lim _{t \rightarrow 0} P_{t}\left(\mathcal{T}_{x}(g)\right)(u)=\mathcal{T}_{x}(g)(u) \geq 0
$$

2ND CASE : We suppose that the function $g$ is in $\mathcal{E}\left(\mathbb{R}^{2}\right)$. Let $\varphi$ be a positive function in $\mathcal{D}\left(\mathbb{R}^{2}\right)$ radial such that supp $\varphi \subset B(0,2), \varphi(y)=1$ for all $y \in B(0,1)$, and for all $y \in \mathbb{R}^{2}, 0 \leq \varphi(y) \leq 1$. We consider the sequence $\left\{\varphi_{n}\right\}_{n \in \mathbb{N} \backslash\{0\}}$ defined by

$$
\forall y \in \mathbb{R}^{2}, \varphi_{n}(y)=\varphi\left(\frac{y}{n}\right) .
$$

As the function $g \varphi_{n}$ belongs to $\mathcal{D}\left(\mathbb{R}^{2}\right)$, then from the first case, we have

$$
\forall x, u \in \mathbb{R}^{2}, \mathcal{T}_{x}\left(g \varphi_{n}\right)(x) \geq 0 .
$$

We obtain (8.1) by using (8.3) and the fact that the sequence $\left\{g \varphi_{n}\right\}_{n \in \mathbb{N} \backslash\{0\}}$ converges to the function $g$ in $\mathcal{E}\left(\mathbb{R}^{2}\right)$.

Corollary 8.2. For all positive function $f$ in $\mathcal{D}\left(\mathbb{R}^{2}\right)\left(\right.$ resp. $\mathcal{S}_{2}\left(\mathbb{R}^{2}\right)$ ), we have

$$
\forall x, y \in \mathbb{R}^{2},{ }^{t} \mathcal{T}_{x}(f)(y) \geq 0 .
$$

Proof. We deduce (8.4) from the relation (5.6) and Theorem 8.1.

TheOREM 8.3. There exists a $\sigma$-algebra $\mathfrak{m}$ in $\mathbb{R}^{2}$ which contains all Borel sets in $\mathbb{R}^{2}$, and for each $x, u \in \mathbb{R}^{2}$, there exists a unique positive measure $m_{x, u}$ on $\mathbb{R}^{2}$ with compact support such that for every $g$ in $\mathcal{E}\left(\mathbb{R}^{2}\right)$, we have

$$
\mathcal{T}_{x}(g)(u)=\int_{\mathbb{R}^{2}} g(z) d m_{x, u}(z) .
$$

Proof. We obtain the results from the relation (8.1) and Theorem 2.14, of [4] p.42.

REMARK 8.4. By using the relations (5.6), (8.5), we obtain for all $f$ in $\mathcal{D}\left(\mathbb{R}^{2}\right)$ and $u, y \in \mathbb{R}^{2}$ the following integral representation of the function ${ }^{t} \mathcal{T}_{u}(f)(y)$ :

$$
{ }^{t} \mathcal{T}_{u}(f)(y)=\int_{\mathbb{R}^{2}} f(z) d \check{m}_{y,-u}(z) .
$$


where $\breve{m}_{y,-u}$ is the positive measure on $\mathbb{R}^{2}$ given by

$$
\int_{\mathbb{R}^{2}} f(z) d \breve{m}_{y,-u}(z)=\int_{\mathbb{R}^{2}} \breve{f}(z) d m_{y,-u}(z), \quad f \in \mathcal{E}\left(\mathbb{R}^{2}\right) .
$$

Corollary 8.5. The product formula (5.3) can also be written in the form

$$
\forall x, u \in \mathbb{R}^{2}, \forall \lambda \in \mathbb{C}^{2}, G_{\lambda}(x) G_{\lambda}(u)=\int_{\mathbb{R}^{2}} G_{\lambda}(z) d m_{x, u}(z) .
$$

Proof. We deduce the relation (8.8) from Theorem 8.3 and the fact that for all $\lambda \in \mathbb{C}^{2}$, the function $G_{\lambda}(x)$ belongs to $\mathcal{E}\left(\mathbb{R}^{2}\right)$.

Corollary 8.6. For all $x, u \in \mathbb{R}^{2}$, we have

$$
\left\|m_{x, u}\right\|=m_{x, u}(1)=1 .
$$

Proof. From the relations (2.9), (2.13) the function $f(x)=G_{-i \rho}(x)$ is the unique solution satisfying $f(0)=1$ of the system

$$
\frac{\partial}{\partial x_{j}} f(x)+\sum_{\alpha \in \mathcal{R}_{+}} \frac{k(\alpha) \alpha^{j}}{1-e^{-\langle\alpha, x\rangle}}\left\{f(x)-f\left(r_{\alpha} x\right)\right\}=0, \quad j=1,2, x \in \mathbb{R}^{2} .
$$

But the constant function $f(x)=1$ is also a solution of this system. Thus

$$
\forall x \in \mathbb{R}^{2}, G_{-i \rho}(x)=1 .
$$

we obtain (8.9) by taking $\lambda=-i \rho$ in the relation (8.8) and by using (8.10).

Corollary 8.7. The Cherednik-Opdam kernel $G_{\lambda}(x)$ admits the following estimate

$$
\forall x \in \mathbb{R}^{2}, \forall \lambda \in \mathbb{R}^{2},\left|G_{\lambda}(x)\right| \leq 1 .
$$

Proof. From the relations (8.8), (2.18), for all $\lambda \in \mathbb{R}^{2}$, we have

$$
\left(\sup _{x \in \mathbb{R}^{2}}\left|G_{\lambda}(x)\right|\right)\left(\sup _{u \in \mathbb{R}^{2}}\left|G_{\lambda}(u)\right|\right) \leq \int_{\mathbb{R}^{2}}\left(\sup _{z \in \mathbb{R}^{2}}\left|G_{\lambda}(z)\right|\right) d m_{x, u}(z) .
$$

Thus by applying (8.9) we get

$$
\sup _{x \in \mathbb{R}^{2}}\left|G_{\lambda}(x)\right| \leq \int_{\mathbb{R}^{d}} d m_{x, u}(z)=1 .
$$

We deduce (8.11) from this relation. 
Proposition 8.8. For all $x, u \in \mathbb{R}^{2}$, we have

$$
\operatorname{suppm}_{x, u} \subset\left\{z \in \mathbb{R}^{2} ;|\|x\|-\|u\|| \leq\|z\| \leq\|x\|+\|u\|\right\} .
$$

To prove this Proposition we need the following Lemma.

Lemma 8.9. Let $x, u \in \mathbb{R}^{2}$ and $f$ be in $\mathcal{D}\left(\mathbb{R}^{2}\right)$. if the support of $f$ is contained in the closed ball $B(0,|\|x\|-\|u\||)$, we have

$$
\mathcal{T}_{x}(f)(u)=0 .
$$

Proof. Set $\eta=|\|x\|-\|u\||$. By the relation

$$
\mathcal{T}_{x}(f)(y)=\mathcal{T}_{y}(f)(x),
$$

we may suppose that $\|x\| \leq\|u\|$. Let $y=\eta+\|x\| \sigma$, with $\sigma$ in the unit sphere $S^{1}$ of $\mathbb{R}^{2}$.

From (5.6), (5.10), we deduce that

$$
\mathcal{T}_{x}(f)(y)=0 .
$$

By replacing $\eta$ by its value in $y$, we obtain $y=\|u\| \sigma$. Thus

$$
\mathcal{T}_{x}(f)(u)=0 \text {. }
$$

\section{Proof of Proposition 8.8}

For all $x, u \in \mathbb{R}^{2}$, the relation (8.8) can also be written in the form

$$
\forall \lambda \in \mathbb{C}^{2}, \mathcal{H}\left(m_{x, u}\right)(\lambda)=G_{\lambda}(x) G_{\lambda}(u),
$$

where $\mathcal{H}$ is the hypergeometric Fourier transform of the measure $m_{x, u}$ which is of compact support.

On the other hand from the relation $(2.22),(2.19)$ there exists a positive constant $M_{0}$ such that

$$
\forall \lambda \in \mathbb{C}^{d},\left|G_{\lambda}(x) G_{\lambda}(u)\right| \leq M_{0} e^{(\|x\|+\|u\|)\|\operatorname{Im} \lambda\|},
$$

and thus (8.14) implies

$$
\forall \lambda \in \mathbb{C}^{2},\left|\mathcal{H}\left(m_{x, u}\right)(\lambda)\right| \leq M_{0} e^{(\|x\|+\|u\|)\|\operatorname{Im} \lambda\|} .
$$

From this relation and Theorem 2.4 of [7], we deduce that

$$
\operatorname{supp}_{x, u} \subset B(0,\|x\|+\|u\|) .
$$

We obtain (8.12) from Lemma 8.9 and (8.15). 


\section{Positivity of the hypergeometric translation operator and its dual associated with the Heckman-Opdam theory}

We consider the trigonometric Dunkl intertwining operator $V_{k}^{W}$ and its dual ${ }^{t} V_{k}^{W}$ associated with the Heckman-Opdam theory, defined respectively on the spaces $\mathcal{E}\left(\mathbb{R}^{2}\right)^{W}$ and $\mathcal{D}\left(\mathbb{R}^{2}\right)^{W}$ (resp. $\left.\mathcal{S}_{2}\left(\mathbb{R}^{2}\right)^{W}\right)$ and signposted to Remark 3.1 (see also [8]).

The hypergeometric translation operator $\mathcal{T}_{x}^{W}, x \in \mathbb{R}^{2}$, associated with the Heckman-Opdam theory, is defined on $\mathcal{E}\left(\mathbb{R}^{2}\right)^{W}$ by

$$
\forall y \in \mathbb{R}^{2}, \mathcal{T}_{x}^{W}(f)(y)=\left(V_{k}^{W}\right)_{x}\left(V_{k}^{W}\right)_{y}\left[\left(V_{k}^{W}\right)^{-1}(x+y)\right]
$$

(see $[8])$.

The dual ${ }^{t} \mathcal{T}_{x}^{W}, x \in \mathbb{R}^{2}$, of the operator $\mathcal{T}_{x}^{W}, x \in \mathbb{R}^{2}$, is defined on $\mathcal{D}\left(\mathbb{R}^{2}\right)^{W}$ (resp. $\left.\mathcal{S}_{2}\left(\mathbb{R}^{2}\right)^{W}\right)$ by

$$
\forall y \in \mathbb{R}^{2},{ }^{t} \mathcal{T}_{x}^{W}(f)(y)=\mathcal{T}_{y}^{W}(f)(-x) .
$$

The main properties satisfied by the operator $\mathcal{T}_{x}^{W}, x \in \mathbb{R}^{2}$, are the following.

1. For all $x \in \mathbb{R}^{2}$, the operator $\mathcal{T}_{x}^{W}$ is continuous from

- $\mathcal{D}\left(\mathbb{R}^{2}\right)^{W}$ into itself.

- $\mathcal{S}_{2}\left(\mathbb{R}^{2}\right)^{W}$ into itself.

2. For all $x, y \in \mathbb{R}^{2}$ and $\lambda \in \mathbb{C}^{2}$, we have the product formula

$$
\mathcal{T}_{x}\left(F_{\lambda}\right)(y)=F_{\lambda}(x) F_{\lambda}(y) .
$$

for the Heckman-Opdam kernel $F_{\lambda}(x)$ defined by (2.14).

3. For all $f$ in $\mathcal{D}\left(\mathbb{R}^{2}\right)^{W}$ (resp. $\mathcal{S}_{2}\left(\mathbb{R}^{2}\right)^{W}$ ) and $x, y \in \mathbb{R}^{2}$, we have

$$
\mathcal{T}_{x}^{W}(f)(y)=\int_{\mathbb{R}^{2}} F_{\lambda}(x) F_{\lambda}(-y) \mathcal{H}^{W}(f)(\lambda) \mathcal{C}_{k}^{W}(\lambda) d \lambda,
$$

where

$$
\mathcal{C}_{k}^{W}(\lambda)=c\left|C_{k}(\lambda)\right|^{-2},
$$

with $c$ is a normalizing constant, $\left|C_{k}(\lambda)\right|^{-2}$ the function given by (4.4), (4.5), and $\mathcal{H}^{W}$ is the hypergeometric Fourier transform associated with the Heckman-Opdam theory defined on $\mathcal{D}\left(\mathbb{R}^{2}\right)^{W}\left(\operatorname{resp} . \mathcal{S}_{2}\left(\mathbb{R}^{2}\right)\right)$ by

$$
\forall \lambda \in \mathbb{R}^{2}, \mathcal{H}^{W}(f)(\lambda)=\int_{\mathbb{R}^{2}} f(x) F_{\lambda}(x) \mathcal{A}_{k}(x) d x .
$$

By using the results of section 8, we deduce the following results. 
Theorem 9.1. For all $x, u \in \mathbb{R}^{2}$ and $g$ in $\mathcal{E}\left(\mathbb{R}^{2}\right)^{W}$ we have

$$
\mathcal{T}_{x}^{W}(g)(x)=\int_{\mathbb{R}^{2}} g(z) d m_{x, u}^{W}(z)
$$

with

$$
m_{x, u}^{W}=\frac{1}{|W|^{2}} \sum_{w, w^{\prime} \in W} m_{w x, w^{\prime} u},
$$

where $m_{x, u}$ is the measure given by (8.5).

Proposition 9.2.

i) The product formula (9.3) can also be written in the form

$$
\forall x, u \in \mathbb{R}^{2}, \forall \lambda \in \mathbb{C}^{2}, F_{\lambda}(x) F_{\lambda}(u)=\int_{\mathbb{R}^{2}} F_{\lambda}(z) d m_{x, u}^{W}(z) .
$$

ii) For all $x, u \in \mathbb{R}^{2}$, we have

$$
\left\|m_{x, u}^{W}\right\|=m_{x, u}^{W}(1)=1 .
$$

iii) For all $x, u \in \mathbb{R}^{2}$, we have

$$
\operatorname{suppm}_{x, u}^{W} \subset\left\{z \in \mathbb{R}^{2} ;|\|x\|-\|u\|| \leq\|z\| \leq\|x\|+\|u\|\right\} .
$$

Proposition 9.3. The Heckman-Opdam hypergeometric function $F_{\lambda}(x)$ admits the following estimate

$$
\forall x \in \mathbb{R}^{2}, \forall \lambda \in \mathbb{R}^{2},\left|F_{\lambda}(x)\right| \leq 1 .
$$

\section{Acknowledgments}

The author is grateful to the referees and the Professor Gwany Hui Kim for the Interest they showed in this paper and for their constructive comments.

\section{References}

[1] I. Cherednik, A unification of Knizhnik-Zamslodchnikov equations and Dunkl operators via affine Hecke algebras, Invent. Math. 106 (1990), 411-432.

[2] G. J. Heckman and E. M. Opdam, Root systems and hypergeometric functions I, Compos Math. 64 (1987), 329-352.

[3] E. M. Opdam, Harmonic analysis for certain representations of graded Hecke algebras, Acta Math. 175 (1995), 75-121.

[4] W. Rudin, Real and complex analysis. Second Edition, McGraw-Hill Book Company, New York, London, Sydney, Tokyo, 1974.

[5] B. Schapira, Contribution to the hypergeometric function theory of Heckman and Opdam : sharp estimates, Schwartz spaces, heat kernel, Geom. Funct. Anal. 18 (2008), 222-250. 
[6] K. Trimèche, The trigonometric Dunkl intertwining operator and its dual associated with the Cherednik operators and the Heckman-Opdam theory, Adv. Pure Appl. Math. 1 (2010), 293-323.

[7] K. Trimèche, Harmonic analysis associated with the Cherednik operators and the Heckam-Opdam theory, Adv. Pure Appl. Math. 2 (2011), 23-46.

[8] K. Trimèche, Hypergeometric convolution structure on $L^{p}$-spaces and Applications, for the Heckman-Opdam theory, Preprint. Faculty of Sciences of Tunis. 2012.

[9] K. Trimèche, Positivity of the hypergeometric translation operators associated with the Cherednik operator, and of the Dunkl translation operators in the one dimentional case, Preprint. Faculty of Sciences of Tunis. 2013.

[10] K. Trimèche, The positivity of the hypergeometric translation operator and of its dual associated with the Cherednik operators and the Heckman-Opdam theory on $\mathbb{R}^{d}$, Preprint. Faculty of Sciences of Tunis. 2013.

[11] K. Trimèche, The positivity of the transmutation operators associated with the Cherednik operators attached to the root system of type $B_{2}$ and $C_{2}$, Preprint. Faculty of Sciences of Tunis. 2014.

Khalifa Trimèche

Department of Mathematics

Faculty of Sciences of Tunis

University of Tunis El Manar

Tunis 2092, Tunisia

E-mail: khlifa.trimeche@fst.rnu.tn 